\title{
Exposure to birch pollen and the risk of allergic and asthmatic manifestations
}

\author{
Timo Hugg ${ }^{1}$, Qianlai Luo ${ }^{1}$, Taina Lajunen ${ }^{1}$, Maritta Jaakkola ${ }^{1}$, and Jouni Jaakkola ${ }^{1}$ \\ ${ }^{1}$ Oulun yliopisto
}

November 24, 2021

\begin{abstract}
This is a Letter and does not include an abstract.

Prof. Jouni J.K. Jaakkola (Manuscript ID:ALL-2021-01267)

To the Editor

Exposure to birch pollen and the risk of allergic and asthmatic manifestations
\end{abstract}

A short running title: Birch pollen exposure and allergic manifestations

Keywords: Allergy, asthma, personal exposure, pollen, regional exposure

Birches are the most dominant pollen type in Northern Europe and have a major role in the development of pollen-related allergic and/or asthmatic symptoms in Northern Europe. ${ }^{1-3}$ Little is known about potential effects of daily personal pollen exposure on daily allergic and asthmatic manifestations. ${ }^{4}$ This is the first study that utilizes personal monitoring in exposure assessment and investigates exposure to birch pollen in relation to pattern of allergic and/or asthma symptoms (including timing, sequence, duration, and intensity).

We assessed potential relations between short-term (daily) exposure to birch pollen and manifestations of allergies and asthma among adults who already had allergic rhinitis and/or asthma. We hypothesized that: 1) Exposure to the peak of pollen concentration is followed by an increase in allergic and/or asthmatic manifestations, 2) A certain biologically plausible sequence can be detected in the occurrence of allergic and/or asthmatic manifestations, 3) A typical duration of allergic and/or asthmatic manifestations can be detected and this depends on the order in which these symptoms occur, and 4) Exposure to the peak of pollen concentration impairs the allergy and asthma control among subjects who have these diseases.

We conducted a panel study among the Espoo cohort members between April 18 and May 24, 2014, in the Helsinki Metropolitan Area during birch pollen season. ${ }^{5-6}$ A total of 19 participants who had allergic rhinitis $(\mathrm{N}=19)$ and/or asthma $(\mathrm{N}=16)$ monitored their daily personal pollen exposure and registered their daily symptoms. Daily allergy and asthma control, inquired in daily diary, were categorized as follows: $1=$ Good, $2=$ Somewhat under control, $3=$ Poorly under control, $4=$ Not at all under control. (see extended Methods, online supplementary material).

The mean daily regional pollen concentrations varied between 18 and 27435 and the mean daily personal pollen exposure varied between 20 and 16773 pollen grains per cubic meter of air during the follow-up period.

Both asthma and allergy control started to decline after people were exposed to pollen peak. Allergy control deteriorated on average $0.268(95 \% \mathrm{CI} 0.087-0.449 ; \mathrm{P}=.004)$ and $0.038(-0.062-0.138 ; \mathrm{P}=.46)$ units when exposed to abundant levels of pollen, and further $0.358(0.184-0.532 ;<.001)$ and $0.096(-0.012-0.204 ; \mathrm{P}=$ 
.08) units when exposed to extremely abundant levels of pollen compared to controls in regional and personal monitoring. (Table 1). Similar trends, although statistically nonsignificant, were detected in asthma control.

Allergy symptom score reached its peak (highest value) simultaneously with the highest pollen peak and was found to follow the trend of pollen exposure more closely. In contrast, asthma symptom score peak occurred with a short lag after the highest pollen concentration and remained at a reduced level during the entire follow-up period. Furthermore, it peaked later after a few additional minor pollen peaks (Figure 1). Most of the participants reported that their symptoms peaked after the regional and the personal pollen peak (Exact binomial test: $\mathrm{P}$ values varied between .059 and $<.001$ ) (Table S1). The nasal system reached its peak first after the peak day of pollen concentration (range in average symptoms peak day 13.1-14.8), followed by throat (14.2-15.9), skin (14.5-15.1), and eye systems (15.4-16.2). Lower respiratory symptoms needed the longest induction period (17.8-18.3) before their peak took place (Table S2).

Worsening of both allergy and asthma symptoms started concurrently with the first peak of birch pollen. Both allergy and asthma control were poorest on the day with abundant and extremely abundant pollen concentrations, but then followed different patterns thereafter. We showed for the first time that there is a biologically plausible sequential order in the occurrence of symptoms after the high pollen exposure.

\section{References}

1. D'Amato G, Cecchi L, Bonini S, et al. Allergenic pollen and pollen allergy in Europe. Allergy 2007;62(9):976-990.

2. Smith M, Jäger S, Berger U, et al. Geographic and temporal variations in pollen exposure across Europe. Allergy 2014;69(7):913-923.

3. Biedermann T, Winther L, Till SJ, Panzner P, Knulst A, Valovirta E. Birch pollen allergy in Europe. Allergy 2019;74(7):1237-1248.

4. Myszkowska D, Bilo B, Stepalska D, Wolek J, Obtulowicz K. Personal and stationary pollen monitoring with regard to pollen allergy symptoms.Allergy Clin Immunol Int 2007;19:108-111.

5. Jaakkola JJ, Hwang BF, Jaakkola N. Home dampness and molds, parental atopy, and asthma in childhood: a six-year population-based cohort study. Environ Health Perspect2005;113(3):357-361.

6. Rantala AK, Jaakkola MS, Mäkikyrö EM, Hugg TT, Jaakkola JJ. Early respiratory infections and the development of asthma in the first 27 years of life. Am J Epidemiol 2015;182(7):615-623.

Authors: Timo T. Hugg, $\mathrm{PhD}^{\mathrm{a}, \mathrm{b}, \mathrm{c}}$, Qianlai Luo, $\mathrm{PhD}^{\mathrm{a}, \mathrm{b}}$, Taina K. Lajunen, $\mathrm{PhD}^{\mathrm{a}, \mathrm{b}, \mathrm{c}}$, Maritta S. Jaakkola, $\mathrm{MD}^{\mathrm{a}, \mathrm{b}, \mathrm{c}}$, Jouni J.K. Jaakkola, MD, $\mathrm{PhD}^{\mathrm{a}, \mathrm{b}, \mathrm{c}, \mathrm{d} *}$

Timo T. Hugg, timo.hugg@oulu.fi; Qianlai Luo, qianlaimo@gmail.com; Taina K. Lajunen, taina.lajunen@oulu.fi, Maritta S. Jaakkola, maritta.jaakkola@oulu.fi, Jouni J.K. Jaakkola,jouni.jaakkola@oulu.fi

${ }^{a}$ Center for Environmental and Respiratory Health Research, P.O.Box 5000 (Aapistie 5B), FIN-90014 University of Oulu, Oulu, Finland

b Biocenter Oulu, University of Oulu, Oulu, P.O.Box 5000 (Aapistie 5A), FIN-90014 University of Oulu, Oulu, Finland

${ }^{\mathrm{c}}$ Medical Research Center Oulu, Oulu University Hospital and University of Oulu, P.O.Box 5000 (Aapistie 5A), FIN-90014 University of Oulu, Oulu, Finland

d Finnish Meteorological Institute, Helsinki, Erik Palménin aukio 1, FIN-00560 Helsinki, Finland

* Correspondence: Professor Jouni J.K. Jaakkola, Center for Environmental and Respiratory Health Research (CERH), University of Oulu, P.O. Box 5000 (Aapistie 5 B), 90014 Oulu, Finland; Tel.:+358406720927, E-mail address:jouni.jaakkola@oulu.fi.

\section{Acknowledgements}


We would like to thank all the volunteers who participated in the study. We also thank the Aerobiology Unit of the University of Turku for lending the Burkard samplers. We thank Dr. Behzad Heibati and Dr. Mari Kauhaniemi (FMI) for their assistance in processing the map images.

This work was supported by the Research Council for Health, the Academy of Finland [grant numbers 266314, 267675, and 267995 (APTA Consortium) and 310372 (GLORIA Consortium)], European Union ECHO civil protection mechanism (EXTREMA, grant number 783180) and the University of Oulu Strategic Funding. The funders had no role in study design, data collection or analysis, decision to publish, or preparation of the manuscript.

\section{Conflict of interest}

All authors declare that they have no conflicts of interest.

TABLE 1 Results on the relations between pollen concentrations and allergy $(n=19)$ and asthma $\left(n=16^{+}\right)$ control.Regression coefficients $(\beta)$ indicate the adjusted differences in the mean allergy and asthma scores between high exposure days (abundant and extremely abundant) and reference days (moderate)

\begin{tabular}{lll}
\hline & Regional pollen exposure $^{++}$ & Regional pollen exposure $^{++}$ \\
\hline Allergy & $\beta$ & $95 \%$ CI \\
Pollen Concentration Moderate (reference) & & \\
Abundant & 0.268 & 0.087 \\
Extremely Abundant & 0.358 & 0.184 \\
Town (ref. = Helsinki) & 0.467 & -0.039 \\
Sex (ref. = male) & -0.020 & -0.544 \\
Flu day (ref. = no flu day) & -0.125 & -0.301 \\
Time variable (ref. = prior to regional pollen peak) & -0.007 & -0.011 \\
Constant & 0.627 & -0.513 \\
\hline
\end{tabular}

\begin{tabular}{lll}
\hline & Regional pollen exposure $^{++}$ & Regional pollen exposure $^{++}$ \\
\hline Asthma & $\beta$ & $95 \% \mathrm{CI}$ \\
Pollen Concentration Moderate (reference) & & \\
Abundant & 0.058 & -0.116 \\
Extremely Abundant & 0.116 & -0.051 \\
Town (ref. = Helsinki) & 0.328 & -0.348 \\
Sex (ref. = male) & 0.271 & -0.427 \\
Flu day (ref. = no flu day) & 0.327 & 0.170 \\
Time variable (ref. = prior to regional pollen peak) & 0.012 & 0.008 \\
Constant & 0.200 & -1.164 \\
\hline
\end{tabular}

${ }^{+}$The number of subjects was 16 because of incomplete data (1 person), lack of diagnosed asthma and asthmatic symptoms (2 persons) and reported flu at the beginning of the study period ( 1 person). ${ }^{++}$Adjusted for town, sex, flu day, and the number of days since pollen peak as time variable. ${ }^{*} \mathrm{P}<.05,{ }^{* *} \mathrm{P}<.01,{ }^{* * *}$ $\mathrm{P}<.001$

\section{Figure legends}

FIGURE 1 Daily average allergy and asthma symptom score levels in relation to the mean daily personal and regional pollen exposures. The number of days on the $\mathrm{X}$-axis refers to the length of follow-up period, which started on the first day of pollen monitoring 


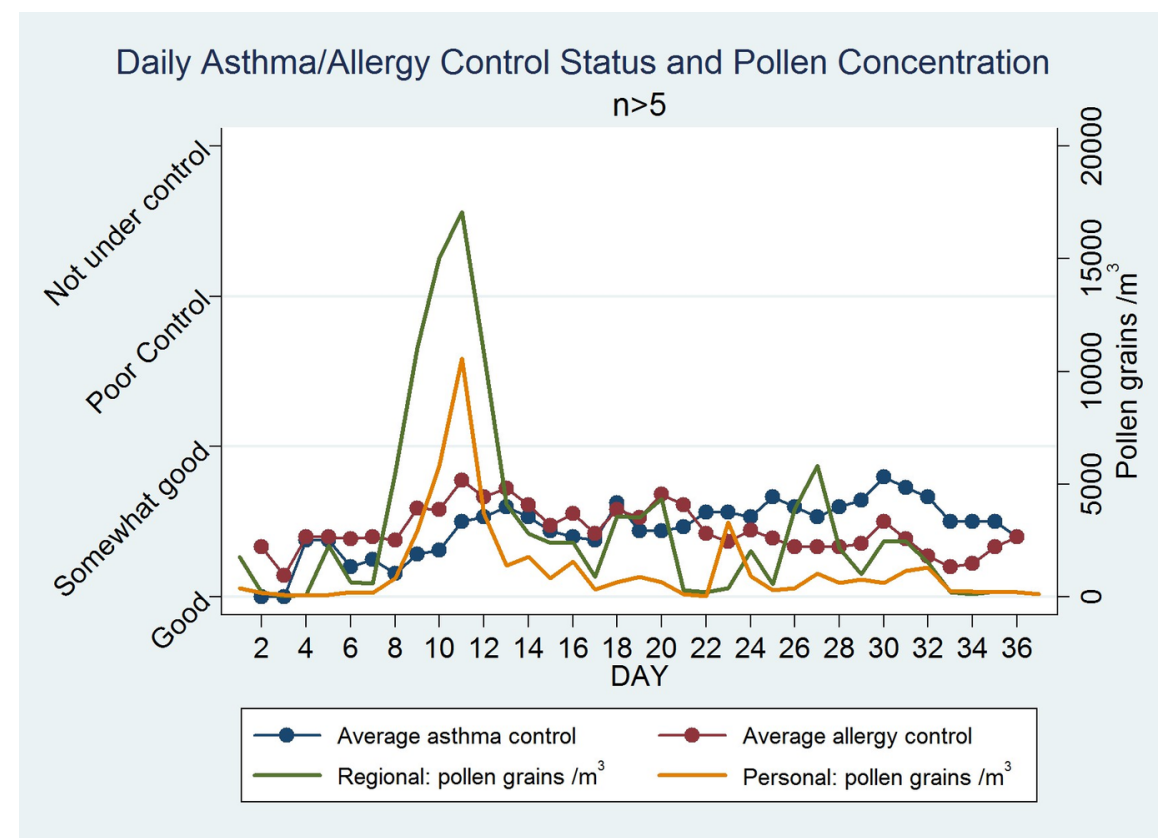

